\title{
finreportr: Financial Data from U.S. Securities and Exchange Commission
}

DOI: $10.21105 /$ joss.00119

\section{Software}

- Review ¿

- Repository u

- Archive ${ }^{\boldsymbol{T}}$

\section{Licence}

Authors of JOSS papers retain copyright and release the work under a Creative Commons Attribution 4.0 International License (CC-BY).

\section{Seward Lee}

\section{Summary}

'finreportr' is an R package ( $\mathrm{R}$ Core Team 2016) that contributes to empirical finance research by automating the download of data relating to the health and transactions of publicly-listed companies, thus simplifying the workflow of financial analysts. It downloads and processes XBRL documents containing financial data from the U.S. Securities and Exchange Commission's EDGAR database. Using a suite of simple functions, data from the Statement of Operations, Balance Sheet, and Statement of Cash Flow of Form 10-Ks may be downloaded, processed, and returned to the user in $\mathrm{R}$ dataframes.

The design of functions included in 'finreportr' is inspired by the quantitative finance package 'quantmod' (Ryan, Ulrich, and Thielen 2016). XBRL-processing capabilities of 'finreportr' are mainly powered by the package 'XBRL' (Bertolusso and Kimmel 2016) under the hood.

\section{References}

Bertolusso, Roberto, and Marek Kimmel. 2016. XBRL: Extraction of Business Financial Information from 'Xbrl' Documents. https://CRAN.R-project.org/package=XBRL.

R Core Team. 2016. R: A Language and Environment for Statistical Computing. Vienna, Austria: R Foundation for Statistical Computing. https://www.R-project.org/.

Ryan, Jeffrey A., Joshua M. Ulrich, and Wouter Thielen. 2016. Quantmod: Quantitative Finacial Modelling Framework. https://CRAN.R-project.org/package=quantmod. 